\title{
Gauging the impact of the COVID-19 pandemic on tuberculosis services: a global study
}

\begin{abstract}
To the Editor:
Copyright (CThe authors 2021.

This version is distributed under the terms of the Creative Commons Attribution Non-Commercial Licence 4.0. For commercial reproduction rights and permissions contact permissions@ersnet.org

Received: 24 June 2021 Accepted: 29 July 2021

\section{○@®@}

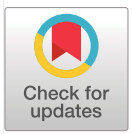

The effects of the coronavirus disease 2019 (COVID-19) pandemic on tuberculosis (TB) disease and TB services emerged in the beginning of 2020 [1, 2]. Epidemiological and clinical studies, including mortality rates of the first cohort of patients with COVID-19 and TB co-infection were described [3, 4]. Several reports from individual countries suggested that the COVID-19 pandemic significantly affected TB services [5-9], including validation by modelling studies [10]. The Global Tuberculosis Network (GTN) reported that the COVID-19 pandemic affected TB services in 33 TB centres from 16 countries in the first 4 months of 2020 [11]. An increased use of telehealth during the COVID-19 pandemic was observed in some TB centres [11]. The major limitations of that study were the short period of observation (January to April 2020 compared to the same period in 2019) and the limited number of variables analysed [11-14].

The current study aims to describe the effects of the COVID-19 pandemic on TB services and TB-related activities during the entire first year of the pandemic, 2020, compared to 2019.

Invitations were sent to the centres previously involved [11], with the addition of Virginia in the USA, Lithuania, Oman and Paraguay. State-wide and/or regional data were collected from Australia (Victoria), Oman, Paraguay, Portugal (Northern Portugal), Russia (Moscow and Arkangelsk Region/Oblast) and USA (Virginia). The coordinating centre and the participating centres had ethics clearance in abidance with their institutional regulations [11]. Data was collected from 1 January 2019 to 31 December 2020.

The following variables were collected monthly: total number of TB disease cases in patients with a new diagnosis or a recurrence; number of newly diagnosed TB disease cases managed in outpatient clinics; number of TB patients discharged from hospital; number of drug-resistant TB cases; number of new TB infections; number of tests performed to diagnose TB infection encompassing tuberculin skin test and interferon- $\gamma$ release assays; number of TB deaths; telehealth services provided for TB disease management (i.e. video directly observed therapy (DOT) or face-to-face teleconsultation). Data quality checks were performed in dialogue with the participating centres under the guidance of two methodology experts.

As TB centres from Moscow and Paraguay only provided TB infection tests and newly diagnosed TB infections on an annual basis, these data were excluded from the monthly data analysis. Details of lockdown and other social restrictions were collected, including dates and whether they were fully or partially implemented (data not shown). Mean \pm sD were computed per month for each year. Analysis was performed using Mann-Whitney U-test and a p-value of $<0.05$ was deemed statistically significant. All computations were performed using Graphpad Prism 7 (version 7.04, GraphPad Software)

43 TB centres located in 19 countries from five continents provided epidemiological data (figure 1a, individual country data not shown). Eight TB centres were enrolled in Spain, six in Oman, five in Italy, three in Brazil, four in Niger, two in Mexico, two in Russia, two in Australia, two in Philippines, and one from each of the nine other countries. Data on TB disease and infection were provided by all centres, except India, the Netherlands and Niger, which did not have information on the number of TB-infected individuals and diagnostic tests provided.

\section{Shareable abstract (@ERSpublications)}

This global study of 43 TB centres from 19 countries demonstrates the impact of COVID-19 pandemic on TB services. Newly diagnosed TB disease, drug-resistant TB, TB deaths, outpatient clinic attendances and newly diagnosed TB infection were reduced. https://bit.ly/3sdHbfk

Cite this article as: Migliori GB, Thong PM, Alffenaar J-W, et al. Gauging the impact of the COVID-19 pandemic on tuberculosis services: a global study. Eur Respir J 2021; 58: 2101786 [DOI: 10.1183/ 13993003.01786-2021].
\end{abstract}


a)

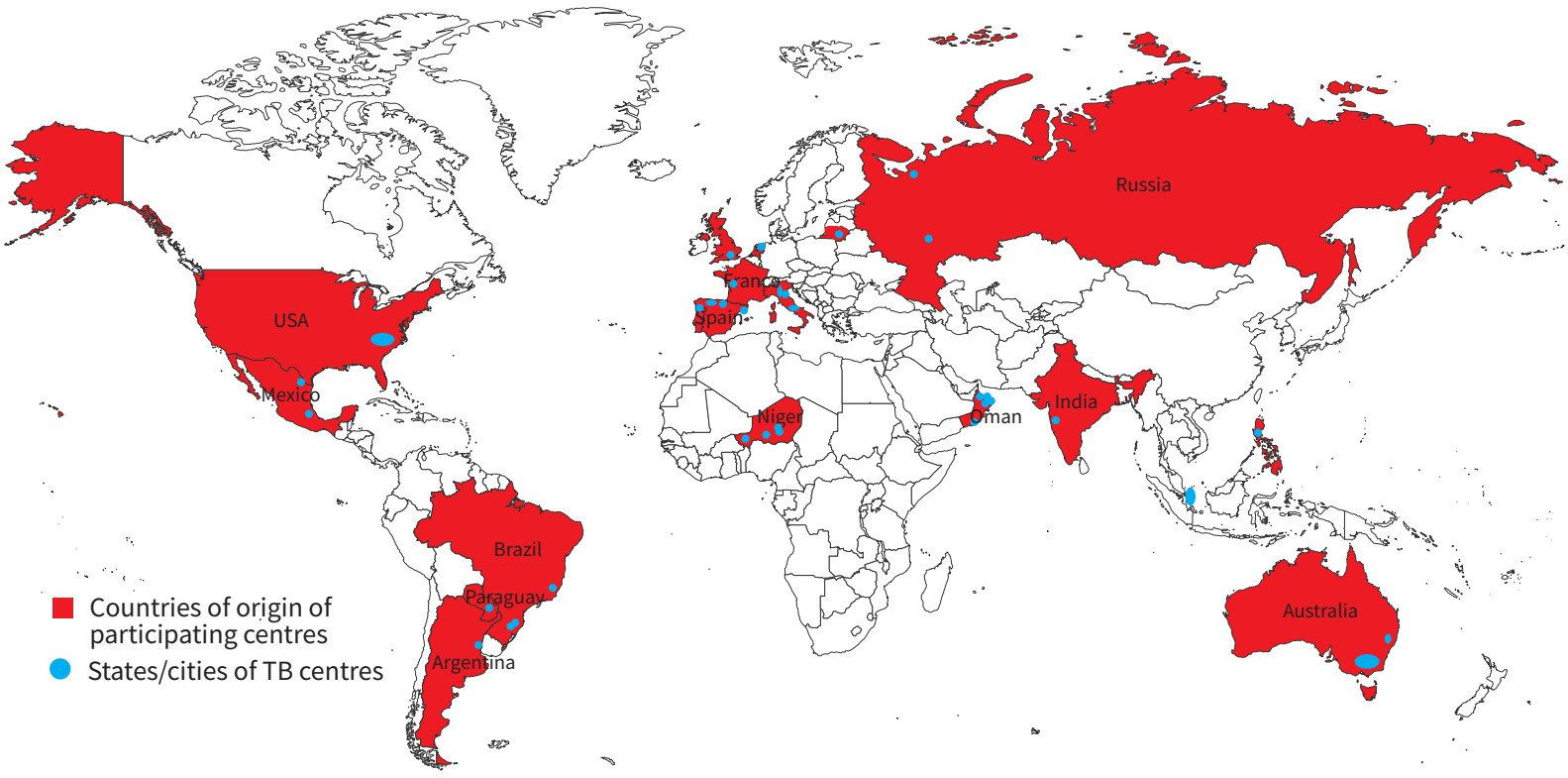

b)

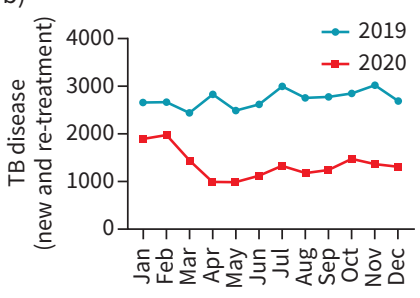

d)

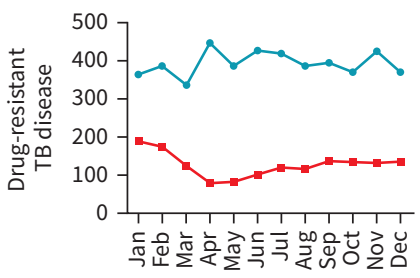

c)

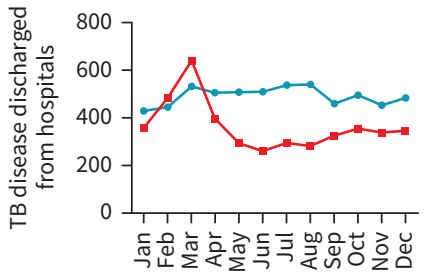

e)

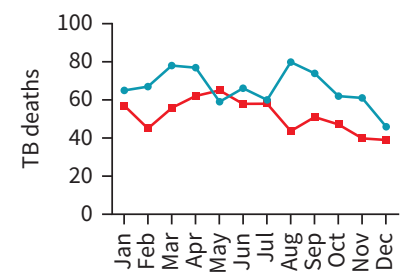

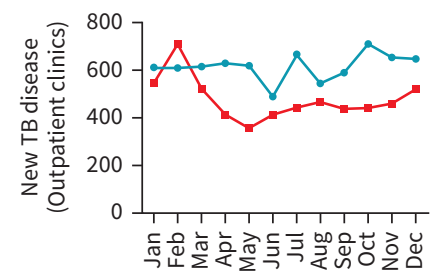

h)

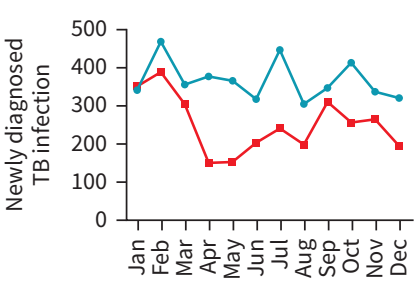

g)

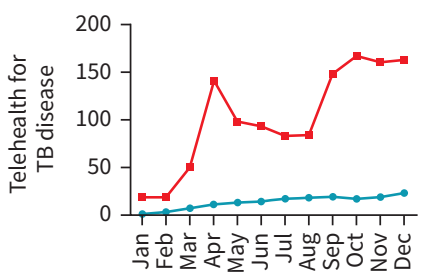

i)

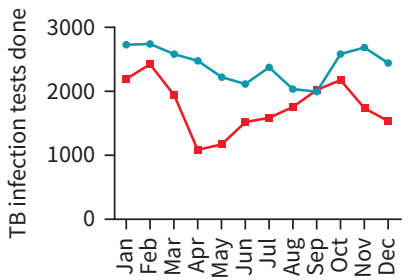

FIGURE 1 a) Countries of the participating centres of the study. State-wide and/or regional data were collected from Australia (Victoria), Oman, Paraguay, Portugal (Northern Portugal), Russia (Moscow and Arkangelsk Region/Oblast) and USA (Virginia). b-i) Tuberculosis (TB) disease and infection trends in 2019 and 2020. b) TB disease; c) TB disease discharged from hospital; d) drug-resistant TB disease diagnosed; e) TB deaths; f) newly diagnosed TB disease in outpatient clinics; g) telehealth use for TB disease in outpatient clinics; h) newly diagnosed TB infection across centres; i) latent TB tests comprising of tuberculin skin tests and interferon- $\gamma$ release assays performed. Data from Moscow and Paraguay are excluded from $\mathrm{h}$ ) and i) as only annual data are available from these two centres.

Lockdowns were implemented in all countries at different times. The earliest lockdown was on 1 February 2020, in Australia, and the latest on 7 April, in Singapore. The majority of the countries implemented multiple lockdowns with partial or full reopening.

TB disease decreased from 32898 (mean \pm SD $2742 \pm 177$ per month) in 2019 to 16396 (1366 \pm 308 per month; $\mathrm{p}<0.0001$ ) in 2020 with a sudden decline in March 2020, concomitantly with the commencement of lockdown in majority of the countries (figure $1 \mathrm{~b}$ ). This epidemiological change was observed in all countries, except the TB centres in Australia, Singapore and Virginia (USA). The number of patients with TB disease discharged from hospitals increased in February and March 2020 compared to the same period in 2019, before a drastic drop commenced in April 2020 (figure 1c) (492 \pm 37 per month in 2019 versus 365 \pm 105 per month in 2020; $\mathrm{p}=0.0007)$. Only two TB centres, located in Australia and Virginia (USA), showed a modest increase.

The number of drug-resistant TB disease cases decreased from 4717 in 2019 to 1527 in 2020 with the decrease starting in March and April 2020 (figure 1d) (393 \pm 31 per month in 2019 versus $127 \pm 32$ per 
month in 2020; $\mathrm{p}<0.001$ ). Although the number of drug-resistant TB cases in some countries was small, an evident decline was observed in Argentina, Brazil, India, Mexico, and Russia. Similarly, the overall TB deaths decreased from 795 in 2019 to 622 in 2020, but subsequently increased in May 2020 (figure 1e) (66 \pm 9 per month in 2019 versus $52 \pm 9$ per month in 2020; $\mathrm{p}=0.0006$ ).

Newly diagnosed TB disease in outpatient clinics reduced from 7364 in 2019 to 5703 in 2020, with a significant decline in March 2020, except the centres in Australia and Virginia (USA) (figure 1f) (613 \pm 57 per month in 2019 versus $475 \pm 90$ per month in 2020; p=0.0005). Despite substantially lower number of newly diagnosed TB disease cases in outpatient clinics during the pandemic year, the number of telehealth activities was much higher in 2020, with two peaks in April and September 2020 (figure 1g) (13 \pm 7 per month in 2019 versus 102 \pm 54 per month in 2020; $\mathrm{p}<0.0001$ ).

Fewer individuals were diagnosed with TB infection, with a decrease in April 2020 (figure 1h) (363 \pm 51 per month in 2019 versus $248 \pm 76$ per month; $\mathrm{p}=0.0007$ ). There were fewer tests performed globally in 2020, with two major troughs in April 2020 and in November/December 2020 (figure 1i) (2413 \pm 269 per month in 2019 versus $1755 \pm 412$ per month; $\mathrm{p}=0.0002$ ). Centres in the Russian Federation performed more TB infection tests in 2020 compared to 2019, which were done on recommendation by the Russian Department of Health.

The impact of the COVID-19 pandemic on TB services was investigated in 19 countries during 2020 compared with the pre-pandemic year 2019. For the first time, evidence is provided that the overall number of patients with TB disease and drug-resistant TB identified at these centres substantially decreased in the first COVID-19 pandemic year compared to 2019, possibly due to difficult access to TB care, lockdown measures and delayed reporting. A similar trend was observed in the majority of the selected countries, mainly in those with higher TB burden. The peak of hospital discharges in March 2020 may be attributed to the need to make space for COVID-19 patients, while outpatient attendances may have increased in February 2020 due to a surge in outpatient prescriptions to tide patients over subsequent months.

A modest increase in TB notifications occurred in Australia and Virginia (USA) in 2020. Given the advanced health systems in these countries, this is likely due to enhanced surveillance to both TB and COVID-19 [12, 14, 15]. A reduction of identified TB disease cases was observed even in low TB incidence countries (e.g. Italy, France and Spain), which were considerably affected by the COVID-19 pandemic. The reduction of identified drug-resistant TB in countries with considerable burden of disease (e.g. Argentina, Brazil, India, Mexico and Russia) raises concerns of future rebounds.

Although TB deaths in 2020 were lower than in 2019, an increase in May and July 2020 was possibly due to deaths misattributed to COVID-19, although other factors such as under-diagnoses or under-reporting are issues in some centres.

Despite decreased patients with TB disease in outpatient clinics, the use of telehealth services was considerably higher in 2020, driven by COVID-19 distancing measures and in keeping with programmatic innovations to address the challenges during the pandemic [16]. The peaks observed in April and October 2020 were temporally related to the first and the subsequent COVID-19 waves. Newly diagnosed TB infection and TB infection tests were also generally lower in 2020 relative to 2019.

Although the large number of countries and collected variables are strengths of the present study, the heterogeneity of the collected information (e.g. hospital discharges and drug-resistant TB numbers which may themselves be affected by multiple factors), the reliance on individual TB centres and not on TB national programmes, and the under-representation of some geographical areas, e.g. Africa, are limitations. The data that we collected were not available to national TB programmes at the time of data collection and the trends shown here may also be biased by the occurrences in countries with the largest number of observations. However, our observations tie in with other substantive disruptions in TB care and notifications that have been reported by the World Health Organization [17].

In summary, this study showed the severe impact of the COVID-19 pandemic on TB services across many countries. There is an urgent need to re-prioritise resources to manage an expected TB resurgence in future.

Giovanni Battista Migliori $\oplus^{1}$, Pei Min Thong ${ }^{2}$, Jan-Willem Alffenaar ${ }^{3,4,5}$, Justin Denholm ${ }^{6,7}$, Marina Tadolini ${ }^{8,9}$, Fatma Alyaquobi ${ }^{10}$, François-Xavier Blanc $\oplus^{11}$, Danilo Buonsenso $\oplus^{12}$, Jin-Gun Cho $\oplus^{4,5,13}$, Luigi Ruffo Codecasa ${ }^{14}$, Edvardas Danila ${ }^{15}$, Raquel Duarte $\oplus^{16}$, José-María García-García $\oplus^{17}$, Gina Gualano ${ }^{18}$, Adrian Rendon ${ }^{19}$, Denise Rossato Silva ${ }^{20}$, Mahamadou Bassirou Souleymane $\oplus^{21}$, Sai Meng Tham ${ }^{22}$, Tania A. Thomas ${ }^{23}$, Simon Tiberi $\circledast^{24,25}$ 
Zarir F. Udwadia ${ }^{26}$, Delia Goletti $\oplus^{18}$, Rosella Centis $\oplus^{1}$, Lia D'Ambrosio $\odot^{27}$, Giovanni Sotgiu $\oplus^{28}$ and Catherine W.M. Ong $\circledast^{2,22,29}$, for the Global Tuberculosis Network

${ }^{1}$ Istituti Clinici Scientifici Maugeri IRCCS, Tradate, Italy. ${ }^{2}$ Dept of Medicine, Infectious Disease Translational Research Programme, National University of Singapore, Yong Loo Lin School of Medicine, Singapore. ${ }^{3}$ The University of Sydney, Pharmacy School, Sydney, Australia. ${ }^{4}$ Westmead Hospital, Sydney, Australia. ${ }^{5}$ Marie Bashir Institute of Infectious Diseases and Biosecurity, The University of Sydney, Sydney, Australia. ${ }^{6}$ Melbourne Health Victorian Tuberculosis Program, Melbourne, Australia. ${ }^{7}$ Peter Doherty Institute for Infection and Immunity, University of Melbourne, Melbourne, Australia. ${ }^{8}$ Infectious Diseases Unit, IRCCS Azienda Ospedaliero-Universitaria di Bologna, Policlinico di Sant'Orsola, Bologna, Italy. ${ }^{9}$ Dept of Medical and Surgical Sciences, Alma Mater Studiorum University of Bologna, Bologna, Italy. ${ }^{10} \mathrm{~TB}$ and Acute Respiratory Diseases Section, Dept of Communicable Diseases, Directorate General of Disease Surveillance and Control, Ministry of Health, Oman. ${ }^{11}$ Centre Hospitalier Universitaire, Nantes, France. ${ }^{12}$ Dept of Woman and Child Health and Public Health, Fondazione Policlinico Universitario A. Gemelli IRCCS, Rome, Italy. ${ }^{13}$ Parramatta Chest Clinic, Parramatta, Australia. ${ }^{14}$ TB Reference Centre, Villa Marelli Institute, Niguarda Hospital, Milan, Italy. ${ }^{15}$ Clinic of Chest Diseases, Immunology and Allergology, Vilnius University Medical Faculty, Centre of Pulmonology and Allergology, Vilnius University Hospital Santaros Klinikos, Vilnius, Lithuania. ${ }^{16}$ National Reference Centre for MDR-TB, Hospital Centre Vila Nova de Gaia, Dept of Pneumology, Public Health Science and Medical Education Dept, Faculty of Medicine, University of Porto, Porto, Portugal. ${ }^{17}$ Tuberculosis Research Programme (PII-TB) SEPAR, Barcelona, Spain. ${ }^{18}$ National Institute for Infectious Diseases (INMI) 'L. Spallanzani' - IRCCS, Rome, Italy. ${ }^{19}$ Centro de Investigación, Prevención y Tratamiento de Infecciones Respiratorias CIPTIR, University Hospital of Monterrey UANL (Universidad Autonoma de Nuevo Leon) Monterrey, Monterrey, Mexico. ${ }^{20}$ Faculdade de Medicina, Universidade Federal do Rio Grande do Sul - UFRGS, Porto Alegre, Brasil. ${ }^{21}$ Damien Foundation, Niamey, Niger. ${ }^{22}$ Division of Infectious Diseases, Dept of Medicine, National University Hospital, Singapore. ${ }^{23}$ Division of Infectious Diseases and International Health, University of Virginia, Charlottesville, VA, USA. ${ }^{24}$ Blizard Institute, Barts and The London School of Medicine and Dentistry, Queen Mary University, London, UK. ${ }^{25}$ Royal London Hospital, Barts Health National Health Service Trust, London, UK. ${ }^{26}$ P.D. Hinduja National Hospital and Medical research Centre, Mumbai, India. ${ }^{27}$ Public Health Consulting Group, Lugano, Switzerland. ${ }^{28}$ Clinical Epidemiology and Medical Statistics Unit, Dept of Medical, Surgical and Experimental Sciences, University of Sassari, Sassari, Italy. ${ }^{29}$ National University of Singapore Institute for Health Innovation \& Technology (iHealthtech), Singapore.

Corresponding author: Catherine W.M. Ong (catherine_wm_ong@nuhs.edu.sg)

Acknowledgements: The article is part of the activities of the Global Tuberculosis Network (GTN); and of the WHO Collaborating Centre for Tuberculosis and Lung Diseases, Tradate, ITA-80, 2017-2020- GBM/RC/LDA.). We thank the GREPI (Groupe de Recherche et d'Enseignement en Pneumo-Infectiologie) a working group from SPLF (Société de Pneumologie de Langue Française); for gathering information.

Collaborators from the Global Tuberculosis Network (by country): Argentina: Instituto Vaccarezza, (UBA), Buenos Aires: Sandra Inwentarz and Domingo Juan Palmero. Australia: Paramatta Chest Clinic, Paramatta-New South Wales: Evan Ulbright. Belgium: Damien Foundation, Brussels: Alberto Piubello. Brazil: Universidade Federal do Rio de Janeiro, Rio de Janeiro: Fernanda Carvalho de Queiroz Mello; Alvorada Tuberculosis Outpatient Clinic, Alvorada - Rio Grande do Sul: Giovana Rodrigues Pereira. France: Centre Hospitalier Universitaire, Nantes: Valérie Pascale Bernard. India: P.D. Hinduja National Hospital and Medical Research Centre, Mumbai: Samridhi Sharma. Italy: Catholic University of Rome, Rome: Roberto Cauda and Silvia Lamonica; National Institute for Infectious Diseases (INMI) 'L. Spallanzani' IRCCS, Rome: Fabrizio Palmieri; University of Sassari, Sassari: Laura Saderi; Tor Vergata University, Rome: Loredana Sarmati and Mirko Compagno. Mexico: Instituto Nacional De Enfermedades Respiratorias Ismael Cosio Villegas, Mexico City: Marcela Muñoz-Torrico. Niger: National Anti-Tuberculosis Centre, Niamey: Alphazazi Soumana. The Netherlands: University of Groningen, Groningen: Onno Akkerman. Paraguay: Ministerio de Salud Pública y Bienestar Social, Asunción: Sarita Aguirre; National Institute of Respiratory Diseases and the Environment, Asunción: Rosarito Coronel Teixeira. Philippines: University Research Co. LLC, Manila: Marianne Calnan. Portugal: University of Porto, Porto: Rui Seixas. Russian Federation: Moscow Research and Clinical Center for TB Control, Moscow: Elena M. Bogorodskaya and Sergey Borisov; Northern (Arctic) Federal University, Arkhangelsk: Anastasia Kulizhskaya and Andrei Mariandyshev. Spain: Hospital Universitario San Agustín, Avilés: Fernando Álvarez-Navascués and José Antonio Gullón-Blanco; Hospital Universitario Central de Asturias, Oviedo: Marta María García-Clemente; Tuberculosis Research Programme SEPAR, Barcelona: Teresa Rodrigo; Hospital de Cruces, Bilbao: Eva Tabernero. Sultanate of Oman: Ministry of Health Oman, Muscat: Seif Al-Abri and 
Khalsa Al-Thohli. UK: Royal London Hospital of Barts Health National Health Service Trust, London, Blizard Institute, Barts and The London School of Medicine and Dentistry, Queen Mary University, London: Heinke Kunst.

Conflict of interest: G.B. Migliori has nothing to disclose. P.M. Thong has nothing to disclose. J-W. Alffenaar has nothing to disclose. J. Denholm has nothing to disclose. M. Tadolini has nothing to disclose. F. Alyaquobi has nothing to disclose. F-X. Blanc has nothing to disclose. D. Buonsenso has nothing to disclose. J-G. Cho has nothing to disclose. L.R. Codecasa has nothing to disclose. E. Danila has nothing to disclose. R. Duarte has nothing to disclose. J-M. García-García has nothing to disclose. G. Gualano has nothing to disclose. A. Rendon has nothing to disclose. D.R. Silva has nothing to disclose. M.B. Souleymane has nothing to disclose. S.M. Tham has nothing to disclose. T.A. Thomas has nothing to disclose. S. Tiberi has nothing to disclose. Z.F. Udwadia has nothing to disclose. D. Goletti has nothing to disclose. R. Centis has nothing to disclose. L. D'Ambrosio has nothing to disclose. G. Sotgiu has nothing to disclose. C.W.M. Ong reports grants from National Medical Research Council (CSAINV17nov014), personal fees (young investigator award) from Institut Merieux, during the conduct of the study; other (honorarium) from Qiagen, outside the submitted work.

Support statement: Part of the work was supported by Ricerca Corrente (Linea 1 and Linea 34 and COVID research) GR-2018-12367178, GR-2016-02364014, Progetto 19. Delibera 257/21. Research reported here was supported in part by the National Center For Advancing Translational Sciences of the National Institutes of Health under award number UL1TR003015. The content is solely the responsibility of the authors and does not necessarily represent the official views of the National Institutes of Health. C.W.M. Ong is supported by Singapore National Medical Research Council (CSAINV17nov014) and recipient of the young investigator award, Institut Merieux, Lyon, France. Funding information for this article has been deposited with the Crossref Funder Registry.

\section{References}

1 Dara M, Sotgiu G, Reichler MR, et al. New diseases and old threats: lessons from tuberculosis for the COVID-19 response. Int J Tuberc Lung Dis 2020; 24: 544-545.

2 Alagna R, Besozzi G, Codecasa LR, et al. Celebrating World Tuberculosis Day at the time of COVID-19. Eur Respir J 2020; 55: 2000650.

3 Tadolini M, Codecasa LR, Garcia-Garcia JM, et al. Active tuberculosis, sequelae and COVID-19 co-infection: first cohort of 49 cases. Eur Respir J 2020; 56: 2001398.

4 Motta I, Centis R, D’Ambrosio L, et al. Tuberculosis, COVID-19 and migrants: preliminary analysis of deaths occurring in 69 patients from two cohorts. Pulmonology 2020; 26: 233-240.

5 Buonsenso D, lodice F, Sorba Biala J, et al. COVID-19 effects on tuberculosis care in Sierra Leone. Pulmonology 2020; 27: 67-69.

6 Adewole OO. Impact of COVID-19 on TB care: experiences of a treatment centre in Nigeria. Int J Tuberc Lung Dis 2020; 24: 981-982.

7 de Souza CDF, Coutinho HS, Costa MM, et al. Impact of COVID-19 on TB diagnosis in Northeastern Brazil. Int J Tuberc Lung Dis 2020; 24: 1220-1222.

8 Wu Z, Chen J, Xia Z, et al. Impact of the COVID-19 pandemic on the detection of TB in Shanghai, China. Int J Tuberc Lung Dis 2020; 24: 1122-1124.

9 Meneguim AC, Rebello L, Das M, et al. Adapting TB services during the COVID-19 pandemic in Mumbai, India. Int J Tuberc Lung Dis 2020; 24: 1119-1121.

10 Cilloni L, Fu H, Vesga JF, et al. The potential impact of the COVID-19 pandemic on the tuberculosis epidemic a modelling analysis. EClinicalMedicine 2020; 28: 100603.

11 Migliori GB, Thong PM, Akkerman O, et al. Worldwide effects of coronavirus disease pandemic on tuberculosis services, January-April 2020. Emerg Infect Dis 2020; 26: 2709-2712.

12 Migliori GB, Tiberi S, Garcia-Basteiro AL, et al. Tuberculosis and its future in the COVID-19 era: the pulmonology series 2021. Pulmonology 2021; 27: 94-96.

13 Ong CWM, Migliori GB, Raviglione M, et al. Epidemic and pandemic viral infections: impact on tuberculosis and the lung. A consensus by the World Association for Infectious Diseases and Immunological Disorders (WAidid), Global Tuberculosis Network (GTN), and members of the European Society of Clinical Microbiology and Infectious Diseases Study Group for Mycobacterial Infections (ESGMYC). Eur Respir J 2020; 56: 2001727.

14 Akkerman O, Aleksa A, Alffenaar JW, et al. Surveillance of adverse events in the treatment of drug-resistant tuberculosis: a global feasibility study. Int J Infect Dis 2019; 83: 72-76.

15 McQuaid CF, Vassall A, Cohen T, et al. The impact of COVID-19 on tuberculosis burden: a review of the data. Int J Tuberc Lung Dis 2021; 25: 436-446.

16 World Health Organization. Programmatic Innovations to Address Challenges in Tuberculosis Prevention and Care During the COVID-19 Pandemic. Geneva, WHO, 2021.

17 World Health Organization. Impact of the COVID-19 Pandemic on TB Detection and Mortality in 2020. Date last accessed: 19 July 2021. www.who.int/publications/m/item/impact-of-the-covid-19-pandemic-on-tb-detectionand-mortality-in-2020 